\title{
Treating akathisia with pregabalin - A case report
}

\section{Pedro Cabral Barata ${ }^{1,2}$ \\ https://orcid.org/0000-0002-7202-5886}

\section{MÁRIO J OÃo SANTOS1,2 \\ https://orcid.org/0000-0002-0798-1951}

TERESA M AIA 2,3

https://orcid.org/0000-0002-1059-1495

1 Prof. Dr. Fernando Fonseca Hospital, Amadora, Portugal.

2 Psychiatry Department of Prof. Dr. Fernando Fonseca Hospital, Amadora, Portugal.

3 Head of Psychiatry Department of Prof. Dr. Fernando Fonseca Hospital, Amadora, Portugal.

Received: 12/12/2018 - Accepted: 2/8/2019

DOI: 10.1590/0101-60830000000199

Barata PC et al. / Arch Clin Psychiatry. 2019;46(3):81-2

\section{Introduction}

Akathisia is a movement disorder characterized by a subjective experience of restlessness alongside an urge to move, generating repetitive movements ${ }^{1,2}$. Its clinical presentation can be confusing and vague, making the diagnosis often problematic and posing an important challenge in clinical practice ${ }^{1}$. This challenge stems not only from its symptoms and difficult treatment, but also from the association with aggression and violence, emergence and/or worsening of suicidal ideation, insomnia and exacerbation of the psychotic illness ${ }^{1,2}$.

Akathisia is the most common movement disorder associated with psychotropic medication ${ }^{1}$, primarily antipsychotic drugs, but also occurs as a side effect of selective serotonin reuptake inhibitors ${ }^{2}$. Among antipsychotics, first generation agents have a higher risk of eliciting akathisia than second generation agents ${ }^{1}$.

While neurobiological mechanisms underlying akathisia are still uncertain, dysfunctions of basal ganglia and striatal circuits have been commonly implicated in its development. Gamma-aminobutyric acid and serotonin are the neurotransmitters most specifically linked to this movement disorder. An imbalance between serotonergic/ noradrenergic and dopaminergic neurotransmitter systems has been classically considered as the origin of akathisia. Nonetheless, other theories have been proposed, such as an overstimulation of the locus ceruleus and D2/D3 receptor occupancy variations in the ventral striatum ${ }^{1}$.

Akathisia is usually classified according to the symptom's development timing. As such, and considering consensual scientific entities, there is acute akathisia, subacute/chronic akathisia, tardive akathisia and withdrawal akathisia. The first occurs within a few days to weeks of antipsychotic drug initiation or dosage increase. Chronic akathisia defines an akathisia that has been present for several months. As the name suggests, tardive akathisia arises late (1-3 months), happening not only during the course of neuroleptic treatment, but also after neuroleptic dosage reduction or discontinuation. Withdrawal akathisia happens within 2 weeks of neuroleptic dosage reduction or discontinuation, typically being selflimited and resolving in 6 weeks ${ }^{1}$. As previously stated, the treatment of akathisia can be difficult. Typically, two strategies are defended when dealing with this issue. The first is a change in medication, either reducing the dose of the culprit agent or switching to another agent. The second strategy lies with the addition of anti-akathisia pharmaceuticals. Many drugs have been proposed to this effect, including propranolol, mirtazapine, anticholinergics (Biperiden, Benztropine, Trihexyphenidyl), Mianserine, benzodiazepines, Cyproheptadine, Amantadine and Clonidine ${ }^{1}$. More recently, there has been a case report showing success of Pregabalin in the treatment of akathisia ${ }^{3,4}$. In this paper, we report one other case of akathisia that improved after the introduction of Pregabalin.

\section{Case report}

The patient was a 73-year-old man with an ICD-10 diagnosis of Bipolar Disorder (F31). He had an history of psychiatric consultations as an outpatient in our unit since 2012 and a psychiatric admission in 2014 due to an antidepressant-induced mania (a selective serotonin reuptake inhibitor together with Mirtazapine). He also had an history of a subjective inner restlessness with a frequent wish to move around "for years" (sic; impossible to stipulate the exact duration), but had not been sufficiently disturbing yet, allowing a relatively good quality of life. His usual medication included a first and a second-generation antipsychotic, an anticonvulsant (acting as a mood stabilizer) and a benzodiazepine - check Table 1 for more details.

In 2018, 1 month after an increase in Quetiapine $(250 \mathrm{mg} /$ day to $350 \mathrm{mg} /$ day), he complained of an exacerbation of the aforementioned restlessness, with mental unease, which started to have a significant impact in his daily activities. It was described as being worse at rest and was ameliorated by moving his legs when walking; therefore, he found it very difficult to sit still in one place and would constantly walk about. The symptoms were not only noticed by him, but also by his wife and daughter. The scores on Barnes Akathisia Rating Scale (BARS) were 5 in 9 (for Objective Akathisia, Subjective Awareness of Restlessness and Subjective Distress) and 4 in 5 (considered "Marked Akathisia”, for Global Clinical Assessment of Akathisia). Before a treatment towards akathisia was started, patient's medical history was reviewed, and a complete physical and laboratory screening was carried out - resulting negative.

The reduction of current Quetiapine was not appropriate in the clinical scenario; Cyamemazine withdrawal was not possible due to the patient's severe insomnia and its worsening on previous failed attempts on dose decreases; and Propranolol introduction was not an option because of an existing medical history of bradycardia. An addition of Mirtazapine $15 \mathrm{mg}$ at bedtime was initially tried, but it increased the symptoms and had to be stopped after one week due

Table 1. Usual medication before pregabalin introduction

\begin{tabular}{|l|c|c|c|c|}
\hline & Breakfast & Lunch & Dinner & Bedtime \\
\hline $\begin{array}{l}\text { Quetiapine } 50 \mathrm{mg} \text {, slow } \\
\text { release }\end{array}$ & & & & 1 \\
\hline Quetiapine $300 \mathrm{mg}$ & 1 & 1 & 1 & 1 \\
\hline Bromazepam $3 \mathrm{mg}$ & 1 & & & \\
\hline Lamotrigine $200 \mathrm{mg}$ & & & & $20 \mathrm{mg}$ \\
\hline Cyamemazine $40 \mathrm{mg} / \mathrm{mL}$ & & & & \\
\hline
\end{tabular}


to symptom severity. Subsequently, Pregabalin $150 \mathrm{mg} /$ day $(50 \mathrm{mg}$, three times-a-day) was initiated. Three weeks after Pregabalin was started, the patient was observed in a psychiatry consultation, and an improvement in akathisia was noted, without significant adverse effects related to the new medicine. The patient reported a substantial reduction in his inner restlessness and did not complain about the need to constantly walk around any longer. A reduction in BARS was observed: Objective Akathisia, Subjective Awareness of Restlessness and Subjective Distress went from 5/9 to 2/9; and Global Clinical Assessment of Akathisia went from 4/5 (Marked Akathisia) to $1 / 5$ (Questionable Akathisia). A follow-up psychiatry consultation was scheduled for 6 months later, where the patient was observed and the clinical benefits of Pregabalin were found to be sustained.

\section{Discussion and conclusions}

To our knowledge, this was the second observation that reported a positive effect of Pregabalin on akathisia ${ }^{3,4}$. Nonetheless, some comparisons between our case report and De Berardis' case ought to be emphasised. Both patients had the diagnosis of Bipolar Disorder (our case from ICD-10, and De Berardis' from DSM-IV). Our patient, on the one hand, was a 73-year-old retired male, probably suffered from a low-grade or subclinical akathisia aggravated within the first month of Quetiapine's introduction, while also being medicated with a first-generation neuroleptic. On the other hand, De Berardis' patient was a 42-year-old housewife, apparently had no history of akathisialike symptoms and developed akathisia after the introduction of Aripiprazole, seeking medical attention 8 months after the initiation of such medication. All things considered, we can affirm that our patient possibly represents a case of acute akathisia, whereas De Berardis' may depict a case of chronic akathisia and/or tardive akathisia (as the symptom's development time frame is not clear).

Pregabalin reduces calcium influx through the binding to the alpha-2-delta subunit of the presynaptic neuron's voltagegated calcium channels, consequently decreasing neuronal hyperexcitability5. As such, Pregabalin reduces the release of several neurotransmitters, including serotonin, norepinephrine, glutamate and dopamine $e^{5,6}$, what may explain the efficacy of Pregabalin on akathisia.

The existence of these case reports may justify the implementation of prospective double-blind, placebo-controlled studies in order to evaluate, in a robust manner, the efficacy of Pregabalin in akathisia.

\section{Funding disclosure}

None to declare.

\section{Conflict of interest}

Nothing to declare.

\section{References}

1. Salem H, Nagpal C, Pigott T, Teixeira A. Revisiting antipsychotic-induced akathisia: current issues and prospective challenges. Curr Neuropharmacol. 2017;15(5):789-98.

2. Barnes TRE. The Barnes Akathisia Rating Scale - Revisited. J Psychopharmacol. 2003;17(4):365-70.

3. De Berardis D, Serroni N, Moschetta F, Martinotti G, Di Giannantonio M. Reversal of aripiprazole-induced tardive akathisia by addition of pregabalin. J Neuropsychiatry Clin Neurosci. 2013;25(2):2007-8.

4. Taylor DM, Barnes TRE, Young AH, editors. Akathisia. In: The Maudsley Prescribing Guidelines in Psychiatry. 13th ed. New Jersey: John Wiley and Sons Ltd; 2018. p. 94-7.

5. Taylor CP, Angelotti T, Fauman E. Pharmacology and mechanism of action of pregabalin: The calcium channel $\alpha 2-\delta$ (alpha2-delta) subunit as a target for antiepileptic drug discovery. Epilepsy Res. 2007;73(2):137-50.

6. Garcia-Borregueo D, Larrosa O, Williams A, Albares J, Pascual M, Palacios J, et al. Treatment of restless legs syndrome with pregabalin: a double-blind, placebo-controlled study. Neurology. 2010;74(23) 1897-904. 